the risk of fractures in elderly women: a prospective study [Abstract]. fournal of Bone and Mineral Research 1989;4 (suppl):S171.

9 Ray WA, Downey W, Griffin MR, et al. Long-term use of thiazide diuretics and risk of hip fracture. Lancet 1989;i:687-90.

10 LaCroix AZ, Wienpahl J, White LR, et al. Thiazide diuretic agents and the incidence of hip fracture. $N$ Engl f Med 1990;322:286-90.

11 Transbol I, Christiansen MS, Jensen GF, et al. Thiazide for the postponement of postmenopausal bone loss. Metabolism 1982;31:383-6.
of

12 Heilbrun LK, Kagan A, Nomura A, Wasnich RD. The origins of epidemiologic studies of heart disease, cancer and osteoporosis among Hawaii Japanese. Hawaii Med f 1985;44:294-6.

13 Vogel JM, Cline JW, Harrison JF, et al. Microcomputer based dual energy photon absorptiometric bone mineral analyzer (VCH). IEEE Transaction on Nuclear Science 1979;26:576-82.

14 Johnson NE, Kutch R, Davis J, et al. The role of calcium in preventing bone loss [Abstract]. Fournal of Bone and Mineral Research 1989;4 (suppl 1):S175.
15 Pickleman J, Paloyan E, Forland M, et al. Thiazide induced parathyroid stimulation. Clin Res 1968;16:468.

16 Wilson DR, Honrath U, Sonnenberg H. Thiazide diuretic effect on medullary collecting duct function in the rat. Kidney Int 1983;23:711-6.

17 Woodhall PB, Tisher CC. Response of the distal tubule and cortical collecting duct to vasopressin in the rat. $\mathcal{F}$ Clin Invest 1973;52:3095-108.

18 Parfitt AM. Chlorothiazide-induced hypercalcaemia in juvenile osteoporosis and hyperparathyroidism. $N$ Engl f Med 1969;281:55-9.

19 Pak CYC, Nicar M, Northcutt C. The definition of the mechanism of hypercalciuria is necessary for the treatment of recurrent stone formers. Contrib Nephrol 1982;33:136-51.

20 Brickman AS, Massey SG, Coburn JW. Changes in serum and urinary calcium during treatment with hydrochlorothiazide: studies on mechanisms. f Clin Invest 1972;51:945-54.

(Accepted 20 September 1990)

\title{
Inappropriate use of laboratory services: long term combined approach to modify request patterns
}

\author{
D Bareford, A Hayling
}

Abstract

Objective-To see whether changes in request patterns for haematological tests could be influenced in the long term by information released from a haematology department.

Design-Analysis of request patterns by hospital divisions before and after intervention and of costs of intervention and savings achieved.

Setting-Haematology laboratory of an inner city district general hospital.

Interventions-Monthly release of a comparison of clinicians' workload statistics, issue of on call guidelines, and promulgation of information (by seminars and factsheets) on appropriate use of tests.

Main outcome measures-Request patterns before and after intervention.

Results-During the year after intervention requests fell by at least a fifth, and the reduction persisted over the next two years. The reduction was most pronounced in relation to inpatients within the division of medicine, for whom requests fell from an average of 4.0 per patient in the six months before intervention to 2.9 per patient in the six months after.

Conclusions - A definite and sustained reduction in inappropriate requests for laboratory investigations may be achieved by an ongoing policy of intervention including issuing guidelines and factsheets and holding seminars, but a positive attitude among senior consultant staff is crucial.

\section{Introduction}

There have been many attempts in the past decade to reduce the amount of inappropriate requests for tests received by hospital clinical laboratories (reviewed by Fraser and Woodford'). The apparent increase in inappropriate requests stems from several factors, ${ }^{23}$ including "routine" diagnostic testing ${ }^{4}$ fear of censure by seniors, ${ }^{5.7}$ entertainment of obscure diagnoses by junior medical staff, excessive frequency of repeat tests, ${ }^{8}$ and irrelevant test results stimulating further inappropriate testing. ${ }^{9}$ The gathering momentum of widespread inappropriate testing has been aided by the ever increasing automation of laboratory procedures, ${ }^{10}$ which make individual tests fairly cheap and the effort to control inappropriate requests comparatively expensive.

Attempts to control inappropriate requesting have included rationing tests, redesigning request forms, educating about appropriate tests for various conditions, educating about costs, issuing feedback information, and using protocols.' Many of these strategies failed completely, ${ }^{8112}$ and those that reduced requests were often expensive in time and manpower and had no sustained effect once they were withdrawn. ${ }^{13-16}$ This study was set up to see if a process of providing information and feedback to clinicians might be cost effective and retain long term influence.

\section{Methods}

All requests and "tests" (see below) received over the previous three and half years by the haematology department of an inner city district general hospital serving a catchment population of 262000 were analysed. A request was defined according to the Steering Group on Health Services Information's guidelines as a "pathology investigation on a single specimen or a group of related specimens taken from an individual patient and sent to a laboratory department at one time, regardless of the number of tests forming part of that investigation". ${ }^{17}$ A "test" was defined as a result or group of results obtained from one manipulation of a specimen. Thus several tests may derive from one request.

The laboratory receives requests from the accident and emergency department; in respect of inpatients and outpatients; from other, outlying hospitals; and from general practitioners. Requests from specific sources were analysed in more detail. Total requests by hospital divisions, split into inpatients and outpatients, were analysed on a monthly basis from February 1987 to December 1989 and compared with inpatient activity taken from deaths and discharges and outpatient activity taken from attendances using information from the patient administration system. On call requests were analysed for the divisions of medicine and surgery on a monthly basis from January 1987 to March 1990. Three tests (full blood count, erythrocyte sedimentation rate, and prothrombin time) carried out for the division of medicine were analysed on a monthly basis from September 1986 to January 1990.

Over eight months between October 1987 and July 1988 several interventions were initiated which might have led to more appropriate use of laboratory facilities. The interventions were costed and were as follows.

(1) From October 1987 each consultant received by post a monthly statement of usage of the laboratory by request and usage of three of the most commonly used tests showing his or her results compared with that of other clinicians subdivided by divisions. From July 1988 this was expanded to include the total cost of the request results provided to his or her clinical team.

(2) From February 1987, after agreement with clinical consultants, on call guidelines were issued to junior medical staff and brought to their attention 
during their introductory lecture. These were based on those described by Lester. ${ }^{10}$ Subsequently consultants in the division of medicine received their comparative usage of the on call service on a monthly basis.

(3) Memorandums were circulated to all medical staff on certain topics such as the increasing misuse of coagulation screens and the relevance of the erythrocyte sedimentation rate.

(4) Radioassay requests for vitamin B-12 and folate estimations were scrutinised along with clinical details and any recent blood count. If the request seemed inappropriate it was cancelled and the serum kept for four weeks. The clinician was informed by printed note.

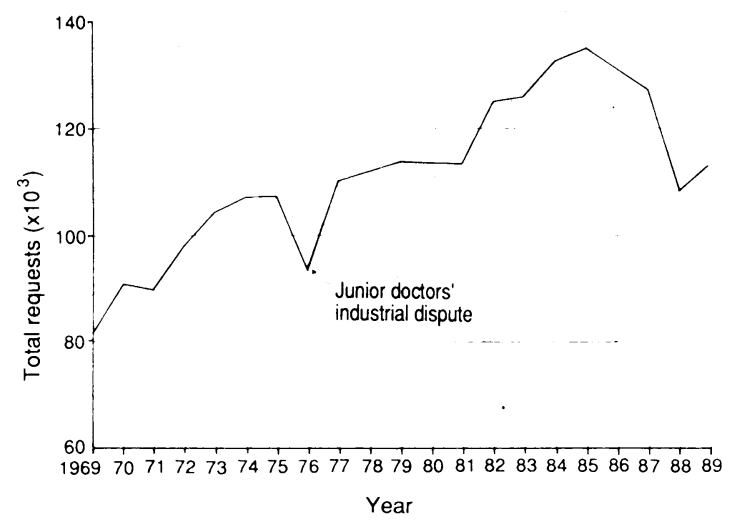

FIG 1-Total requests for haematological tests received yearly during $1969-89$

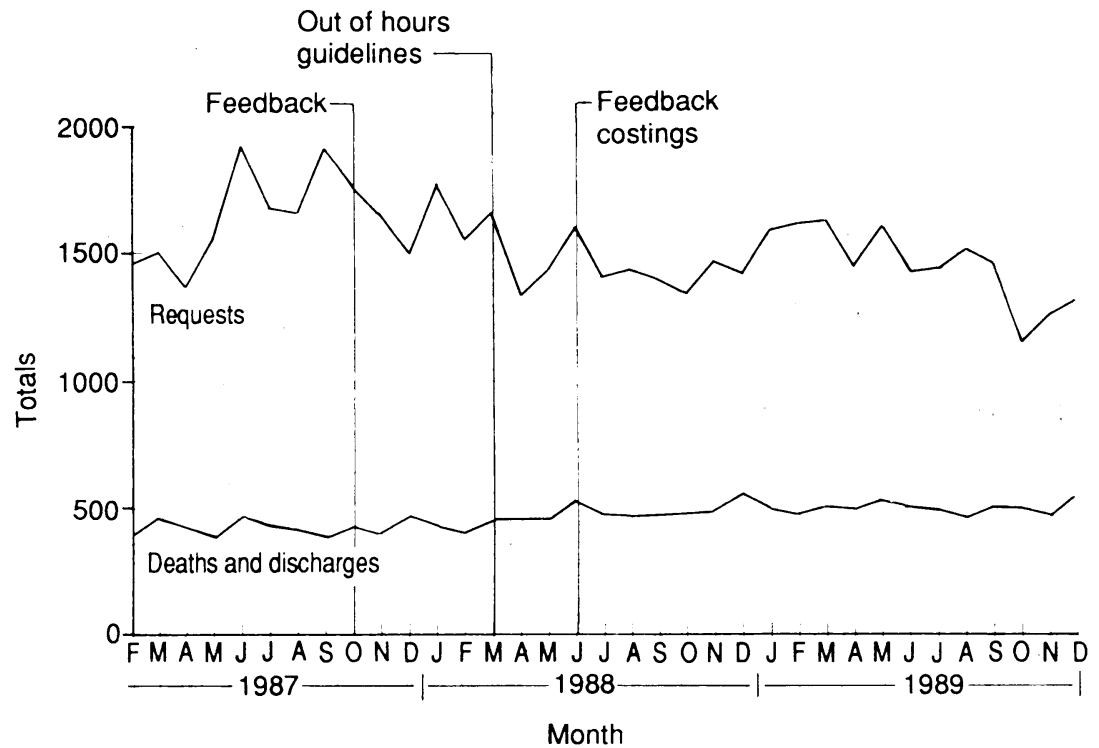

FIG 2-Monthly requests for haematological tests in respect of inpatients in division of medicine compared with deaths and discharges (1987-9)

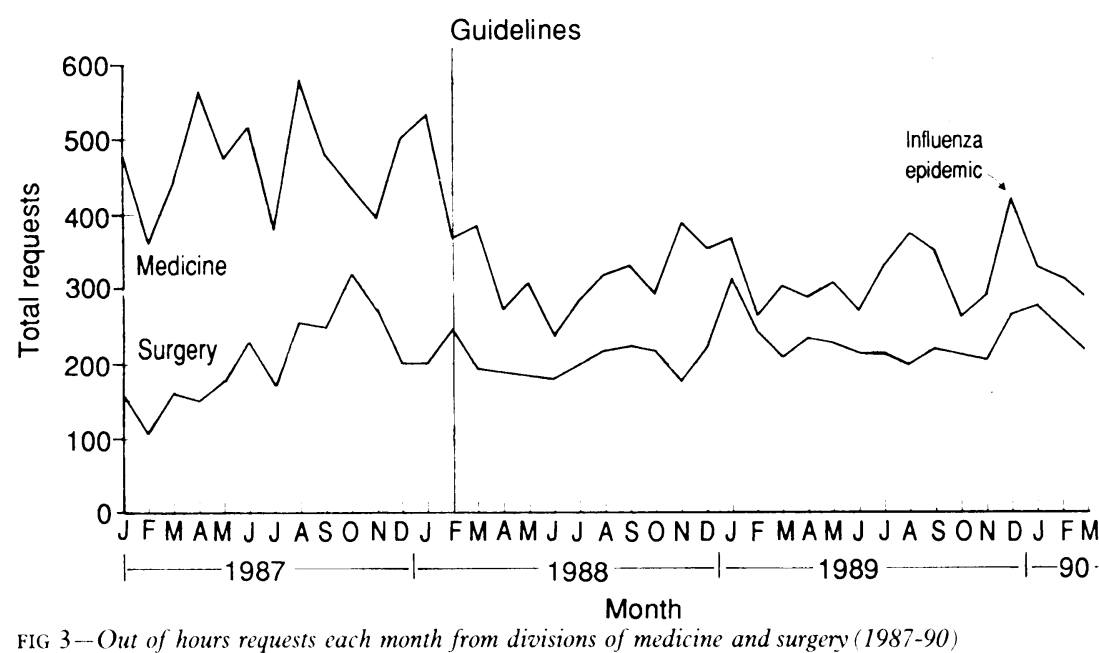

Requests and tests were analysed before and after these interventions to see if there had been any measurable effect and, if so, whether this had persisted in the long term (greater than one year). The cost benefit was compared with the manpower cost in the time and money that it took to institute these interventions and maintain them.

Data analysis was carried out on a DEC Datasystems minicomputer (Digital Equipment Company Ltd, Reading) using MUMPS software running Telepath's pathology system (Telepath Systems Ltd, Birmingham) and on an IBM compatible personal computer using Smartware Version 3.1 (Informix Software Ltd, Middlesex) incorporating the Telepath pathology data management module. The costing method applied was initially used by Tarbit for costing clinical biochemistry services but proved to be transferable for use in haematology. ${ }^{18}$ The method provides a means of apportioning both direct and indirect costs for consumables and labour by individual test and requests. The request can be further broken down into routine and out of hours costs. This method integrated well with the Telepath pathology system.

\section{Results}

There was a decline in total requests from all sources in 1988 (fig 1). This was maintained in 1989 despite the transfer of requests to the laboratory from a peripheral ear, nose, and throat centre. The most striking effect of the interventions was on requests in respect of inpatients in the division of medicine (fig 2). Requests fell from an average of 4.0 per patient in the six months before intervention to an average of 2.9 per patient in the six months after. There was no detectable effect on inpatient requests from the division of surgery, which remained at a ratio of $2 \cdot 7$, nor on those from obstetrics and gynaecology.

A reduction in obstetric and gynaecology outpatient requests from 1650 a month to 1000 a month resulted from an agreement with the division of obstetrics that two blood counts during pregnancy were adequate in normal circumstances rather than testing routinely at each attendance. The outpatient request ratio was unaffected in the divisions of medicine and surgery.

On call requests from the division of medicine fell by $38 \%$ after the introduction of guidelines, and this effect was maintained. There was no detectable effect on requests from the division of surgery (fig 3 ).

Analysis of specific tests within the division of medicine showed a fall in estimations of erythrocyte sedimentation rate over the period and a similar trend in respect of full blood counts and prothrombin times (fig 4). Requests for vitamin B-12 and folate estimations fell by some $20-25 \%$ whereas only $9 \%$ were held back. There was a subsequent demand to analyse specimens withheld in $1 \%$ of cases.

The spreadsheet containing the request patterns of consultants took four hours to set up. The subsequent analysis of costs of each request and test took roughly 80 hours of a mid-grade medical laboratory scientific officer's time. Subsequent data collection and distribution took one and a half hours of a medical laboratory scientific officer's time and one hour of secretarial time a week. Consultant memos (roughly three a year), lectures (about two a year), and scrutinising vitamin B-12 and folate requests took an average of one hour a week. Estimated savings amounted to $£ 18100$ a year (table).

\section{Discussion}

This study has shown a positive and prolonged effect of intervention on influencing requests for hospital haematology laboratory services. The most substantial 


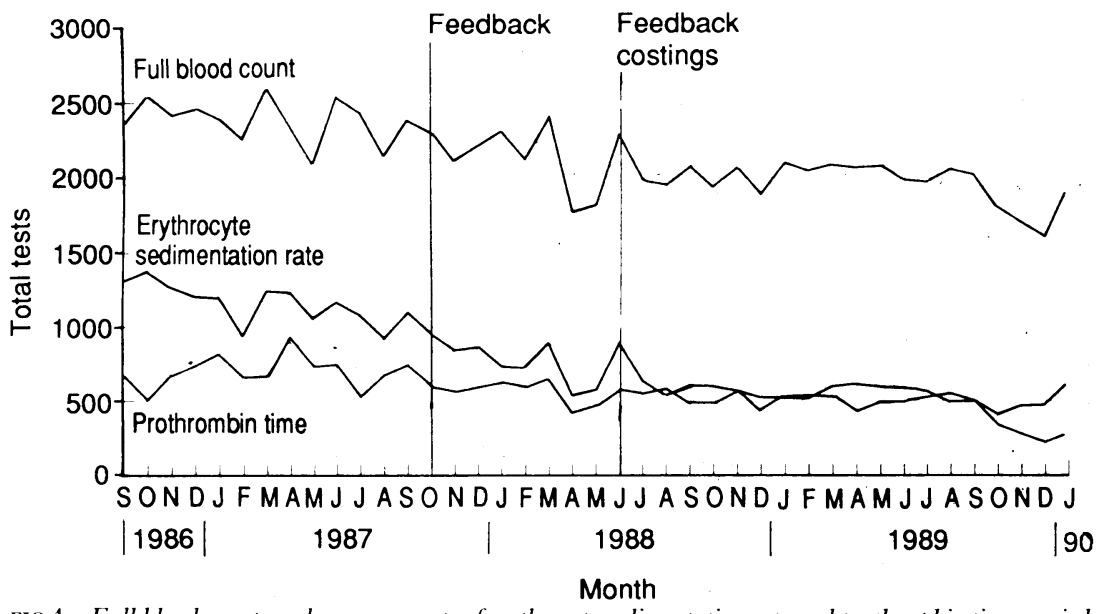

FIG 4-Full blood counts and measurements of erythrocyte sedimentation rate and prothrombin time carried out monthly for division of medicine (1986-90)

Setting up costs, ongoing costs, and estimated savings yearly

\begin{tabular}{lr}
\hline & $£$ \\
\hline Setting up costs: & \\
84 Hours of grade 2 medical laboratory & 604.80 \\
scientific officer time at $£ 7.20 / \mathrm{h}$ & 86.50 \\
5 Hours of consultant time at $£ 17.30 / \mathrm{h}$ & 7.60 \\
2 Hours secretary time at $£ 3.80 / \mathrm{h}$ & 698.90 \\
\hline Total & \\
\hline Ongoing costs/year: & \\
$11 / 2$ Hours of grade 2 medical laboratory & 561.60 \\
$\quad$ scientific officer time/week $\times 52$ & 899.60 \\
1 Hour of consultant time/week $\times 52$ & 197.60 \\
1 Hour of secretary time/week $\times 52$ & 1658.80 \\
\hline Total & \\
\hline Estimated savings/year: & \\
1 Whole time equivalent medical & 10600.00 \\
$\quad$ laboratory scientific officer & \\
Consumables on 15000 requests a year at & 7500.00 \\
$\quad 50$ p/request & 18100.00 \\
\hline Total &
\end{tabular}

response was achieved by the junior medical staff in the division of medicine, the heaviest users of the service, whose level of experience and types of patients seen were leading to more inappropriate use of investigations. Before the issue of guidelines it was estimated that up to $40 \%$ of on call requests may have been inappropriate, which was very close to the $38 \%$ reduction subsequently achieved. This was despite poor attendance by juniors at initial three monthly guideline review meetings and the indication by questionnaire that they rarely consulted the guidelines. The principle of guidelines was brought to the attention of each change of house staff during their introductory lecture.

Total requests in respect of inpatients in the division of medicine also fell. This resulted from an increased awareness by junior clinical staff of audit, not only through memorandums and guidelines but also by virtue of the positive attitudes of their consultant staff, who were issued with the monthly statements. Memorandums were specifically seen to reduce tests of sedimentation rate, which were often coupled with requests for blood counts automatically, and prothrombin and partial thromboplastin time tests, which were often coupled together inappropriatelyfor example, in paracetamol overdosage-when a prothrombin time test would suffice. Other interventions also helped achieve reductions such as a simple change of protocol in the antenatal clinic. The lack of effect on requests from the division of surgery may have been related to the low level of usage, and in respect of outpatients because of appropriate usage by consultant staff initially.

This study may have had some effect because it included a combined approach with education, issue of guidelines, positive feedback, and a continuing process. Other studies have shown some advantages of these individually. Fowkes et al achieved an immediate $64 \%$ reduction in haematological and biochemical requests with the use of guidelines and review of notes. ${ }^{16}$ Active feedback has been shown to work and be more successful than passive feedback, ${ }^{19}$ especially when clinicians are shown comparative data of usage with their peers, ${ }^{1520}$ and long term approaches ${ }^{21}$ have been shown to be more effective than short term studies, ${ }^{13}$ which are often viewed as experimental. This study also coincided with, or maybe was instrumental in, a steady change of senior and junior clinicians' attitudes towards more effective use of hospital resources. This would explain the continued decline in some test requests long after the introduction of intervention. A positive attitude by senior clinicians has been recognised as crucial for the success of any method. ${ }^{22} 23$

The intervention in this study required little in manpower or monetary resources and led not only to financial savings but to more effective use of medical laboratory scientific officers' time. The time and resource spent in educating new junior staff could be circumvented by appropriate instruction at medical school. If topics on resource management and medical audit entered the curriculum future junior staff would already be conscious of the appropriate use of resources. With the impending changes brought about by the government's white paper ${ }^{24}$ there is evidence that present changing attitudes among senior clinicians are beginning to allow previous strategies to succeed, ${ }^{25} 26$ and possibly the time is ripe to try again.

1 Fraser CG, Woodford FP. Strategies to modify the test-requesting patterns of clinicians. Ann Clin Biochem 1987;24:223-31.

2 Connelly D, Steele B. Laboratory utilisation: problems and solutions. Arch Pathol Lab Med 1980;104:59-62.

3 Fraser CG. Interpretation of clinical chemical laboratory data. Oxford: Blackwell Frientific, 1986:2-3.

4 Anonymous. Routine diagnostic testing [Editorial]. Lancet 1989;ii:1190-1.

5 Hardison JE. To be complete. N Engl F Med 1979;303:71-6.

6 Eisenberg JM, Williams SV. Cost containment and changing physicians' practice behavior. Can the fox learn to guard the chicken coop? JAMA 1981;246:2195-201.

7 Wong ET, Lincoln TL. Ready! Fire! . . Aim! An inquiry into laboratory test ordering. JAMA 1983;250:2510-3.

8 Eisenberg JM, Williams SV, Garner L, et al. Computer based audit to detect and correct overutilisation of laboratory tests. Med Care 1977;15:915-21.

9 World Health Organisation Working Group. Assessment of benefits and costs of clinical laboratory testing. Geneva: WHO, 1984. (LAB/84-5.)

10 Lester E. A new strategy for out-of-hours laboratory investigations. Ann Clin Biochem 1986;23:497-500.

11 Wong ET, McCarron MM, Shaw ST. Ordering of laboratory tests in a teaching hospital. FAMA 1983;249:3076-80.

12 Schroeder SA, Myers LP, McPhee SJ, et al. The failure of physical education as a cost containment strategy. $\mathcal{J} A M A$ 1984;253:225-30.

13 Eisenberg JM. An educational program to modify laboratory use by house staff. Journal of Medical Education 1977;52:578-81.

14 Everett GD. Impact of supervision by medical teachers and in-patient test control programmes on the out-patient test utilisation of residents. Med Educ 1985; 19:138-42.

15 Marton KI, Tul V, Sox HC. Modifying test-ordering behavior in the outpatient medical clinic. A controlled trial of two educational interventions. Arch Intern Med 1985; 145:816-21.

16 Fowkes FGR, Hall R, Jones JH, et al. Trial of strategy for reducing the use of laboratory tests. $B M \mathcal{F} 1986 ; 292: 883-5$.

17 Körner E. First report to the secretary of state of the steering group on health services information. London: HMSO, 1984:109.

18 Tarbit IF. Costing clinical biochemistry services as part of an operational management budgeting system. $\mathcal{F}$ Clin Pathol 1986;39:817-27.

19 Mitchell MW, Fowkes FGR. Audit reviewed: does feedback on performance change clinical behaviour? f R Coll Physicians Lond 1985;19:251-4.

20 Schroeder SA, Kenders K, Cooper JK, et al. Use of laboratory tests and pharmaceuticals: variation among physicians and effects of cost audit on pharmaceuticals: variation among physician
subsequent use. $\mathcal{F} A M A$ 1973;225:969-73.

21 Griner PF. The medical house staff, Strong Memorial Hospital. Use of laboratory tests in a teaching hospital; longterm trends. Ann Intern Med 1979;90:243-8.

22 Cohen DJ, Jones P, Tittenberg B, et al. Does cost information availability reduce physician test usage. Med Care 1982;20:280-92.

23 Everett GD. Laboratory utilisation by house staff: the importance of attitude. Clin Res 1982;30:640a.

24 Secretaries of State for Health, Wales, Northern Ireland, and Scotland. Working for patients. London: HMSO, 1989.

25 Feely J, Chan R, Cocoman L, et al. Hospital formularies: need for continuous intervention. $B M \mathcal{F}$ 1990; 300:28-30.

26 Delamothe T. Hey, big spender. BMF 1990;300:286 